\title{
La implementación de RDA en la Argentina \\ Implicancias en las prácticas de organización de la información seguidas por las bibliotecas
}

\author{
Gerardo Salta \\ Universidad de Buenos Aires. Facultad de Filosofía y Letras. Buenos Aires, Argentina I gersalta@gmail.com / \\ https://orcid.org/oooo-0002-9561-961X
}

\begin{abstract}
Resumen
Se describen los principales apartados del proyecto de investigación que trata sobre el proceso de implementación del estándar Recurso, Descripción y Acceso (RDA) en las bibliotecas de la República Argentina. Tal proyecto se dirige a cumplimentar los requisitos del Doctorado en Bibliotecología y Ciencia de la Información de la Facultad de Filosofía y Letras de la Universidad de Buenos Aires. El artículo enfatiza el objeto y la problemática de investigación, el estado de la cuestión-con énfasis en las etapas del proceso de implementación de RDA y la situación de la organización de la información en diferentes lugares del mundo ante la aparición de RDA. Continua con el enunciado del marco teórico, los objetivos, el método y las técnicas a ser aplicadas en la investigación. Concluye con la formulación de la hipótesis y las principales propiedades relacionadas con la unidad de análisis sobre las que se desenvolverá la investigación.
\end{abstract}

RDA implementation in Argentina: implications in organization of information followed by libraries

\begin{abstract}
This article describes a research Project belonging to a doctoral degree of Doctorado en Bibliotecología y Ciencia de la Información of the Facultad de Filosofía y Letras at Universidad de Buenos Aires. The study deals with the standard Resource Description and Access (RDA) implementation process in the Argentine libraries. The work enumerates a research object and problem, a state of the art, which stress RDA implementation stages and a situation of information organization at different places in the world when emerged RDA. It continues with the statement of the theoretical framework, the objectives, the method and the techniques to be applied in the research. It concludes with the formulation of the hypothesis and the main properties related to the unit of analysis on which the investigation will be developed.
\end{abstract}

Palabras clave

$R D A$

Implementación Bibliotecas

Organización de la información Argentina

Keywords

RDA

Implementation stages

Libraries

Organization of information

Argentina 


\section{Introducción}

Las Reglas de Catalogación Angloamericanas, 2a. ed. (en inglés, Anglo American Cataloging Rules, 2nd edition, en adelante AACR2)se publicaron originalmente en 1978, basadas en el estándar marco Descripción Bibliográfica Internacional Normalizada (en inglés, International Standard Bibliographic Description, en adelante ISBD) y elaboradas por la Federación Internacional de Asociaciones de Bibliotecarios y Bibliotecas (en inglés, International Federation of Library Associations and Institutions, en adelante IFLA). Casi veinte años después, las crecientes demandas por la necesidad de reformas sobre AACR2, conducen a la organización de la International Conference on the Principles and Future Development of $A A C R$, en 1997, con la finalidad de tratar las problemáticas emergentes de dicho estándar y considerar las tendencias presentes y futuras respecto a los recursos de información y a la gestión de información en las bibliotecas. Concurrentemente, en 1998, la IFLA publica el modelo Requisitos Funcionales de los Registros Bibliográficos (en inglés, Functional Requirements for Bibliographic Record, en adelante, FRBR), en tanto modelo entidad-relación aplicado a los distintos componentes del universo bibliográfico. En 2017, IFLA publica un nuevo modelo denominado Modelo de Referencia Bibliotecaria (en inglés, Library Reference Model, en adelante LRM) que consolida, reconcilia y subsume la familia de modelos conceptuales FR, compuesta por FRBR, los Requisitos Funcionales de los Datos de Autoridad (en inglés, Functional Requirements for Authority Data, en adelante FRAD) y los Requisitos Funcionales para Datos de Autoridad de Materia (en inglés, Functional Requirements for Subject Authority Data, en adelante FRSAD). Luego de la Reunión de Expertos en Catalogación, realizadas en diferentes partes del mundo entre 2003 y 2007, IFLA disemina los Principios Internacionales de Catalogación (en inglés, International Cataloguing Principles, en adelante PIC) que se publican por primera vez en 2009 y reciben su primera actualización en 2016. El texto enunciado sumariza el contexto previo y los antecedentes en relación al surgimiento del estándar Recurso, Descripción y Acceso (en inglés, Resource Description and Access, en adelante RDA). Esta normativa de descripción y acceso utilizada en la organización de información desarrollada en las bibliotecas constituye el objeto de estudio del proyecto de investigación para aspirar al Doctorado en Bibliotecología y Ciencia de la Información de la Facultad de Filosofía y Letras de la Universidad de Buenos Aires, bajo la dirección de la Magister Elsa Barber (Salta, 2020). El siguiente artículo informa sobre los principales apartados del citado proyecto de investigación, particularmente en lo atinente a la delimitación del objeto, el enunciado del problema de investigación, el estado de la cuestión acerca del proceso de implementación de RDA y la situación de la organización de la información existente en distintos lugares del mundo ante la inminencia de RDA, como es el caso de Nueva Zelanda, Canadá, España y Argentina. Prosigue enunciando el marco teórico, los objetivos, el método y las técnicas de investigación a ser aplicadas. Finalmente, se trazan las hipótesis de trabajo y las principales propiedades relacionadas con la unidad de análisis de la investigación, las que van a representar las variables de los casos bajo estudio.

\section{Objeto de análisis y problema de investigación}

Continuando con el devenir seguido por los códigos de catalogación en el ámbito bibliotecario, enunciado en el apartado introductorio de este artículo, en el año 2004, el Joint Steering Committee for Revision of AACR publica el borrador de un nuevo código de catalogación denominado Anglo American Cataloging Rules, 3th edition (AACR3). En base a los comentarios críticos recibidos, se decide un cambio de rumbo, acordando la conformación de un nuevo estándar de catalogación designado como RDA (Salta, 2016). La versión en inglés de RDA se publica formalmente en línea en la plataforma web RDA Toolkit en 2010, mientras que aparece en formato impreso en 2013. Entre 
junio y diciembre de 2010, Library of Congress (en adelante, LC), National Library of Medicine y National Agricultural Library llevan a cabo el U.S. RDA Test de alcance nacional para todo Estados Unidos. Los resultados de la prueba se presentan en 2011. El 31 de marzo de 2013, LC decide la implementación de RDA. La versión digital en español de RDA se publica en el año 2015, dentro del RDA Toolkit, mientras que la versión impresa en español aparece en el año 2016.

El propio estándar RDA explicita en su pauta 0.3, "Relación con otras normas de descripción y acceso al recurso", como uno de sus objetivos básicos, mantener la compatibilidad con los datos y con las tecnologías bibliotecarias patrimoniales precedentes, como AACR2, MARC21 e ISBD (RDA Toolkit, 2021). En tal sentido, se establece una estrecha transición entre los estándares de contenido y de codificación que dominan el escenario bibliotecario internacional alrededor de los últimos cincuenta años. Esta prolongación, por relación transitiva, transversaliza e influye, en menor o mayor grado, sobre las decisiones y los pasos seguidos en la implementación y aplicación de RDA en la organización de la información llevada a cabo por las bibliotecas alrededor del mundo (El-Sherbini, 2013). El concepto de transición junto con la noción de hibridación de las prácticas, los instrumentos y los productos del control bibliográfico generados por las bibliotecas, se vuelven imprescindibles para interpretar y analizar la coyuntura actual del control bibliográfico. La noción de catálogo híbrido ya era familiar a fines del siglo XIX, en tanto Cutter razona que, si una biblioteca cuenta con un catálogo de fichas e inserta en él fichas sucesivas, por ejemplo, elaboradas y distribuidas por LC, ambos tipos de fichas pueden permanecer juntas en la gaveta sin que los usuarios noten la diferencia (Welsh y Whaite, 2012). En 2011, Library of Congress-Program for Cooperative Cataloging Policy Statements (LC-PCC PS) observa que el ambiente de la catalogación ya connota una naturaleza híbrida. En la misma dirección, Online Computer Library Center (OCLC) incluye en WorldCat metadatos bibliográficos creados con AACR2, RDA y otras normas internacionales, contribuyendo a la conformación de un ambiente de descubrimiento y representación cada vez más diverso y heterogéneo (Welsh y Whaite, 2012).

El problema de investigación trata con el análisis de las características, las modalidades y las vicisitudes particulares que entraña y adquiere el proceso de implementación de RDA en las bibliotecas de la República Argentina, y por extensión, en las bibliotecas latinoamericanas, desde una perspectiva secuencial, metódica y transicional. Contar con una concepción de esta naturaleza implica pensar dicha implementación como una sucesión de pasos o estadios, concatenados e imbricados entre sí, requeridos para lograr un éxito de larga duración con respecto al impacto que el nuevo estándar de catalogación tenga en la organización de la información presente y futura dentro de las bibliotecas. El carácter transicional se denota en concebir que el nuevo estándar RDA está intrínsecamente vinculado con las normas de contenido y codificación - como AACR2 y MARC21 - antes que pensar en un corte abrupto y drástico con las prácticas seguidas en la organización de la información.

\section{Estado de la cuestión}

A comienzos del siglo XXI, muchos catalogadores alrededor del mundo comienzan a percibir la necesidad de una nueva aproximación para la descripción y el acceso a los recursos de información alojados en las bibliotecas. En tal sentido, AACR2 demuestra poseer un manejo bastante complicado y difícil de seguir respecto a los recursos electrónicos y en línea. Asimismo, los expertos en catalogación comienzan a considerar la necesidad imperiosa de una catalogación basada en principios, en lugar del establecimiento de reglas para subsanar cada posible situación que se presente. Es decir, promover el tránsito desde una catalogación legalista - caracterizada por 
contar con una regla adecuada para cada caso y una autoridad que resuelva cualquier problema que se presente - hacia una catalogación pragmática adaptada a la necesidad de tipos particulares de bibliotecas y de usuarios, con reglas simples y un fuerte influjo del juicio del catalogador (Joudrey, Taylor y Miller, 2015).

La IFLA se transforma en un actor central en este devenir a partir de la elaboración y publicación del modelo FRBR, luego subsumido junto con los demás modelos FR bajo LRM, así como de los PIC. El modelo FRBR enumera las entidades encontradas en el universo bibliográfico y las reúne en tres grupos, en base a las funciones o roles que cumplimentan. Asimismo, identifica los atributos o características asociadas con cada entidad, así como también las relaciones que existen al interior y entre los grupos de entidades. Finalmente, el modelo conceptual suscribe diversas tareas que realizan los usuarios al utilizar datos bibliográficos y herramientas de recuperación de información. LRM, por su parte, genera una serie de cambios que afectan a las entidades, a las relaciones entre entidades y a las tareas del usuario, modificando de manera directa la estructura de RDA, en tanto nuevo modelo subyacente. Los PIC constituyen un conjunto común de principios que se ven como necesarios para los usuarios y los catalogadores que interactúan con el catálogo en línea de acceso público (en inglés, Online Public Access Catalog, en adelante OPAC), en tanto sistemas de descubrimiento y búsqueda (Joudrey, Taylor y Miller, 2015).

RDA implica una nueva organización, totalmente diferente a la estructura de áreas y elementos de descripción comportada por AACR2 e ISBD, basándose íntegramente en los modelos conceptuales FR y en los PIC diseñados por IFLA-a los que se alude más arriba. Asimismo, mantiene una estrecha relación con AACR2, erigiéndose en el reemplazo y la continuidad de este estándar anterior, aunque configurándose como más inclusiva que su antecesora. Asimismo, se vincula estrechamente con las estructuras de las actuales bases de datos que relacionan entre sí metadatos bibliográficos y de autoridad. RDA está diseñada y dirigida para interactuar mejor con los recursos digitales, aunque también abarca la descripción y acceso vinculada con recursos de información tradicionales. En tal sentido, el contenido de RDA puede ser utilizado con toda la variedad existente de esquemas de codificación pertenecientes a diferentes comunidades - como Metadata Object Description Standard (MODS), Dublin Core, MARC21, para nombrar solo algunos de ellos. RDA se adecua mejor a las tecnologías de bases de datos emergentes que facilitan a diversas instituciones contar con dispositivos tecnológicos para la recuperación, la captura y el almacenamiento de datos. En tal sentido, el principio subyacente de RDA "tomar lo que se ve", maximiza y favorece estas prácticas tecnológicas. Finalmente, al manifestarse en forma de herramienta en línea en la Web, permite que sus pautas sean adaptadas a perfiles particulares, como la catalogación de tipos especiales de formatos, el agregado de notas locales en línea de parte de los catalogadores y la integración de políticas institucionales para ser consultadas en línea, entre otros factores importantes de mencionar (Joudrey, Taylor y Wisser, 2018; RDA Steering Committee, 2020; RDA Toolkit, 2021).

\section{Los estadios de implementación de RDA}

La implementación de RDA en las bibliotecas comporta un conjunto de estrategias destinadas a facilitar la determinación de cuáles son las necesidades presentes en los pasos de la puesta en vigor del nuevo estándar de catalogación. En consecuencia, hablar de la implementación de RDA implica tener en cuenta, por un lado, un acuerdo tácito y extendido acerca de los tópicos abarcados dentro de la implementación y, por otro lado, un orden de prelación y de sucesión de dichos tópicos en forma de pasos. En base a lo sostenido por El-Sherbini (2013), pueden establecerse las siguientes etapas de implementación de RDA. 


\section{Etapa 1: Familiaridad con RDA y los modelos conceptuales FR}

El primer estadio abarca contenidos vinculados con el propio estándar RDA, así como con los modelos conceptuales FR que le sirven de sustento. Esta etapa se encuentra relacionada con el entrenamiento de RDA, abarcando la familiaridad con los modelos FR y la versión original de RDA, pero también, en la actualidad, tomar en consideración el modelo LRM y la versión oficial de RDA en inglés (RDA Toolkit, 2021). Las modificaciones impuestas por el modelo LRM en la configuración de RDA deberán considerarse y plasmarse en un nuevo reentrenamiento de los catalogadores para utilizar el estándar de descripción y acceso. La familiaridad también comprende los PIC de IFLA, la aprehensión de la terminología y las pautas enunciadas en RDA, las similitudes y diferencias de RDA con AACR2, el mapeo de las pautas de RDA con la codificación establecida por los formatos MARC21 Bibliográfico y de Autoridad y las principales funcionalidades soportadas por el RDA Toolkit.

\section{Etapa 2: Entrenamiento en RDA}

En relación al segundo ítem, dentro de la literatura sobre la implementación de RDA, el entrenamiento fue uno de los aspectos que revistió el mayor foco de interés. El entrenamiento en RDA, engloba la totalidad de los aspectos mencionados bajo el punto anterior, existiendo una profusa bibliografía concentrada en el desarrollo de diversas consideraciones particulares relativas a la capacitación y la formación en el uso del nuevo estándar, siendo uno de los aspectos que reviste el mayor foco de interés en lo relativo a su adopción. Hitchens y Symons (2009) enfatizan el paralelismo que existe entre RDA y AACR2, tanto en los aspectos vinculados con su historia, el vocabulario, la organización, las reglas y las opciones presentes en ambos estándares. Discuten la necesidad de editar los manuales de procedimientos y las hojas de entrada para soportar el cambio de estándar y los aspectos concernientes al desarrollo de programas de entrenamiento en catalogación, dirigidos tanto para el personal de las bibliotecas como a los docentes y alumnos de las escuelas de Bibliotecología y Ciencia de la Información (Hitchens y Symons, 2009). Por otro lado, Kuhagen (2011) discute los preparativos del entrenamiento desarrollado por LC para el U.S. RDA Test, dirigido al personal de LC y de otras bibliotecas que participaron del test. Brinda detalles de los módulos de entrenamiento de RDA, las actividades y las fases de entrenamiento llevadas a cabo. Concluye con una serie de lecciones aprendidas, entre las que se destacan brindar el entrenamiento sobre RDA contando con la disponibilidad y el acceso al estándar de parte de los profesionales e incluir recursos o situaciones conocidas al practicar con el código. Con respecto a la implementación, sostiene la necesidad de pensar que la totalidad de los participantes no se encuentran familiarizados con AACR2, como única vía para conocer RDA. Finalmente, subraya la catalogación por copia o la reutilización de metadatos como operación significativa desarrollada por la mayoría de las bibliotecas que participaron del test y la discusión e ilustración constante a los partícipes de la importancia de un entorno de datos vinculados que subyace a RDA (Kuhagen, 2011).

\section{Etapa 3: Política de catalogación para aplicar con RDA}

El tercer paso consiste en la elaboración de políticas y de procedimientos formalizados de catalogación con RDA de alcance local, enraizados en un contexto coordinado a nivel nacional, que garantice la coherencia en la aplicación e interpretación dentro del país y, por relación transitiva, que favorezca el intercambio fluido de metadatos y el uso compartido de recursos a nivel internacional entre bibliotecas de diferentes lugares del mundo (Oliver, 2010). Las políticas, denominadas actualmente también como buenas o mejores prácticas, tienen una larga tradición y presencia en las bibliotecas. En el caso de RDA se vuelven cruciales e indispensables, en la medida que clarifican, deciden, explican e interpretan las instrucciones del estándar. Las 
políticas de catalogación afectan de manera directa la aceptación o el rechazo de aquellas pautas de RDA calificadas como alternativas y opciones. Las alternativas comportan otra aproximación a la instrucción anterior inmediata, mientras que las opciones ocurren en forma de adición y de omisión. Las políticas también describen la aproximación particular que la biblioteca tiene en relación con los elementos núcleo de RDA y como los catalogadores implementan ciertas pautas del estándar (Joudrey, Taylor y Miller, 2015). En la etiqueta Recursos del RDA Toolkit se permite el acceso a distintas políticas, como la mencionada LC-PCC PS, British Library Policy Standard (BL), National Library of Australia (NLA), Library and Archives Canada (LAC), las pautas de aplicación para los países de lengua alemana (cuyas siglas son D-A-CH AWR) y Best Practices for Music Cataloging Using RDA and MARC21 (MLA BP) (RDA Toolkit, 2021). Asimismo, es importante mencionar que OCLC cuenta con una Declaración de Políticas de RDA, enunciada desde el 31 de marzo de 2013 (OCLC, 2019). Por otro lado, si bien se presenta la posibilidad cierta de que las bibliotecas creen y elaboren sus propias políticas, debe tenerse en cuenta que su diseño y desarrollo debe favorecer la uniformidad de criterios a lo largo del tiempo, requiriendo de una inversión de envergadura tanto de tiempo como de recursos humanos y de dinero. Por esta razón, y muchas otras que este escrito no agota, la gran mayoría de las bibliotecas de diferentes lugares del mundo siguen políticas de catalogación de RDA ya establecidas, como LC-PCC PS (Cronin, 2011; Hanson y Parks, 2013). Una de las primeras cuestiones que una biblioteca necesita preguntarse antes de empezar a utilizar RDA, es si va a aplicar el estándar a un subconjunto de las operaciones de catalogación, o si va a aplicarlo extensivamente en todas las formas de catalogación y en todos los tipos de materiales que colecciona (Cronin, 2011). La mayoría de las experiencias desarrolladas por las bibliotecas que utilizan RDA, sostenidas por la bibliografía, dividieron sus políticas en base a un criterio funcional, estableciendo las decisiones concernientes a la catalogación original escindidas de la catalogación por copia.

Deutsche National Bibliothek - junto con las bibliotecas nacionales de Austria y de la parte de Suiza que habla lengua alemana - conformaron un proyecto de implementación conjunta de RDA a partir de 2012, finalizado en 2015. La primera de las etapas de dicho proyecto, consistió en la elaboración de la política de RDA a aplicarse en los países de lengua alemana, la que condujo a la inserción de las particularidades propias del ambiente cultural y lingüístico alemán en aquellos lugares donde se consideró que RDA comportó pautas inadecuadas o innecesarias, y donde no existió ninguna LC-PCC PS que sea aplicable. Asimismo, se agregaron elementos núcleo adicionales, estableciendo un piso mínimo para la catalogación de los países de lengua alemana. Se alentó el juicio del catalogador, dando libertad a cada biblioteca para describir otros elementos de datos más allá de los definidos en el estándar. En lo atinente al tratamiento de las alternativas y opciones de RDA, antes de decidir su adopción o rechazo, también se consultaron las políticas de las demás bibliotecas nacionales que ofician como miembros del RDA Steering Committee (RSC) con la finalidad de brindar la suficiente uniformidad para que el intercambio de datos se encuentre libre de obstáculos (Behrens, Frodl y Polak-Bennemann, 2014). National Library Board (NLB) de Singapur diseña su política de catalogación con RDA enfatizando las necesidades particulares de la biblioteca al describir un recurso de información, concluyendo en la primacía que reviste el alineamiento estrecho con LC-PCC PS, por encima de lo instruido por RDA. En lo referido a los elementos núcleo, durante el entrenamiento del personal, se brindan precisiones referidas tanto a los elementos núcleo de RDA como a aquellos estipulados por LC-PCC PS (Choi, Yusof e Ibrahim, 2014). Kent State University Library (KSUL), elabora sus políticas de catalogación original, a fines de 2012 y principios de 2013, adoptando LC-PCC PS como base para la toma de decisiones. En tal sentido, elige alinear su política de catalogación con las PCC Core Guidelines, en tanto estas establecen un piso superior de elementos núcleo 
en comparación con LC y RDA. Se incita el juicio de los catalogadores para que agreguen información que consideran necesaria con la finalidad de cumplimentar las tareas del usuario de FRBR presentes en RDA (Maurer y Panchyshyn, 2014). El asunto vinculado con la puntuación ISBD presente en metadatos RDA codificados con MARC21, muestra el alineamiento directo de la totalidad de las bibliotecas nacionales y académicas relevadas en esta ponencia, con lo establecido por LC-PCC PS, que sostiene la asignación de la puntuación ISBD a partir del Ap. D.1 de RDA, y la Declaración de Política de OCLC, que indica no remover la puntuación ISBD de los metadatos maestros (El-Sherbini, 2013; Joudrey, Taylor y Miller, 2015; Maurer y Panchyshyn, 2014; RDA Toolkit, 2021). Es indispensable manifestar, como lo hacen Maurer y Panchyshyn (2014), la necesidad de que las bibliotecas cuenten con una política de catalogación por copia bajo RDA, en la medida que la mayor flexibilidad de las pautas establecidas por este nuevo estándar de descripción y acceso repercute directamente en una menor consistencia de los elementos de datos en los metadatos importados mediante la catalogación derivada de otras bibliotecas. Esta situación puede agregar complejidad a las tareas propias de la catalogación por copia, a la vez que puede incrementar el tiempo destinado a la tarea de editar un metadato con la finalidad de lograr su adecuación y correspondencia con los estándares establecidos a nivel local (Maurer y Panchyshyn, 2014). Finalmente, resta manifestar que las políticas de catalogación tratan con la toma de decisiones relacionada con la continuidad de seguir aceptando metadatos elaborados con AACR2, tal como están o mejorando su calidad mediante el agregado de elementos de datos provenientes de RDA. La formalización y los efectos de esta decisión se encontrarán directamente imbricados con las determinaciones tomadas en las etapas 4 y 5 de implementación venideras.

\section{Etapa 4: Determinación de una fecha de implementación de RDA}

La cuarta fase constituye la verdadera bisagra de la implementación de RDA, marcando el antes y el después de la irrupción del nuevo código de catalogación en la biblioteca. Como lo han establecido gran cantidad de bibliotecas nacionales, bibliotecas académicas y bibliotecas públicas, así como utilidades bibliográficas a lo largo y ancho del mundo, fijar una fecha exacta de implementación de RDA tiene un efecto directo sobre los demás estadios de implementación. A la par del diseño y establecimiento de una política de catalogación, se requiere como decisión obvia que la biblioteca fije una fecha de adopción de RDA, con el consecuente punto de partida en la aceptación y recepción de metadatos bibliográficos elaborados con dicho estándar. La adopción de RDA de parte de LC en Estados Unidos, el 31 de marzo de 2013, inicia el cambio en la curva de la oferta de metadatos bibliográficos y de autoridad RDA para recursos de información en inglés, comenzando a ser factible la aceptación de metadatos RDA por medio de la catalogación por copia (Maurer y Panchyshyn, 2014; Tosaka y Park, 2013). Esta situación se ve reforzada en la medida que OCLC establece dentro de su política de RDA en WorldCat no permitir, desde el 31 de marzo de 2013, trascripciones paralelas en AACR2 y RDA para el mismo recurso de información (OCLC, 2019).

\section{Etapa 5: Divulgación de la implementación de RDA al personal y a los usuarios de la biblioteca}

El quinto estadio trata con los efectos de RDA en la visualización de los metadatos en el OPAC, situación que implica pensar en una notable mejora de la funcionalidad del catálogo de acuerdo a las innovaciones sostenidas por los motores de búsqueda y los modelos FR y con el grado de adaptación que revisten los sistemas integrados de gestión bibliotecaria (SIGB) para alojar las nuevas etiquetas MARC21 y las modificaciones sostenidas por RDA (El-Sherbini, 2013). La inadecuación de MARC21 con respecto a su posicionamiento para poder representar eficazmente el modelo entidad-relación que subyace a RDA, implica diversos escritos e iniciativas clave en pos del desarrollo 
de un novedoso marco de datos. En tal sentido, su funcionamiento integral no puede ser totalmente evaluado hasta que LC complete su trabajo con Bibliographic Framework Transition Initiative (BIBFRAME), dirigido a rediseñar los servicios bibliotecarios y favorecer una mejor adaptación de los metadatos a las necesidades actuales y futuras de la comunidad bibliotecaria (Tosaka y Park, 2013). A la par que se desenvuelve la situación aludida, el objetivo continuidad, bajo 0.4.2.4 de RDA, sostiene mantener la compatibilidad con los datos y con las tecnologías bibliotecarias patrimoniales precedentes. De tal forma, los metadatos bibliográficos elaborados con RDA se presentan de acuerdo a las especificaciones de puntuación prescritas por ISBD y de codificación sostenidas por MARC21, coexistiendo con registros AACR2 en las bases de datos y en los catálogos actuales (Joudrey, Taylor y Miller, 2015; Maurer y Panchyshyn, 2014; Tosaka y Park, 2013).

\section{Etapa 6: Integración o hibridación de metadatos}

La sexta etapa trata con la hibridación de metadatos con elementos RDA y AACR2. Esta situación implica tomar en cuenta la integración de los nuevos metadatos de RDA con los metadatos patrimoniales - es decir, aquellos que no están confeccionados con RDA- almacenados en el SIGB utilizado por la biblioteca. Concatenado a esta acción, se encuentra la alternativa de que la biblioteca decida re-catalogar una porción particular de su colección por medio del uso de las funcionalidades de los sistemas en línea, como en el caso de la aplicación de los cambios y actualizaciones globales provistos por la mayoría de los sistemas automatizados de bibliotecas. Maurer y Panchyshyn (2014) sostienen que el imperativo del principio de economía vuelve inadecuada esta última alternativa. Los metadatos bibliográficos creados con anterioridad a la fecha de implementación de RDA estipulada por la biblioteca, no deberían sufrir ningún cambio hacia RDA. En consecuencia, la re-catalogación de los metadatos bibliográficos producidos con AACR2, no es ni ha sido un factor clave ni principal en la implementación de RDA. Asimismo, es plausible estimar que del total de los costos que entraña la implementación inicial de RDA, aquellos vinculados con la integración o la hibridación de los datos devenidos de RDA en los metadatos bibliográficos preexistentes bajo AACR2, son de menor cuantía en comparación con los que puede entrañar la re-trascripción de cada metadato AACR2 a RDA (Maurer y Panchyshyn, 2014). Kuhagen (2011) subraya catalogación por copia o la reutilización de metadatos como operación significativa desarrollada por la mayoría de las bibliotecas participantes del U.S. RDA Test y la discusión e ilustración constante a los participantes de la importancia de un entorno de datos vinculados subyacente a $\mathrm{RDA}$. Esta situación pone de relieve la naturaleza híbrida del catálogo de la biblioteca, producto de la concurrencia de metadatos bibliográficos pre-AACR, AACR, AACR2 y, ahora, RDA. En consecuencia, la decisión política que debe tomarse involucra considerar si hibridizar o no los metadatos descriptivos patrimoniales elaborados con AACR2, u otros estándares, para asimilarlos cada vez más a RDA. Los beneficios de esta acción consisten en tender hacia la uniformidad provista por un único estándar dentro del catálogo, que facilite la gestión de los datos, a la vez que permita a los usuarios contar con metadatos más consistentes y útiles (El-Sherbini, 2013; Maurer y Panchyshyn, 2014; Tosaka y Park, 2013).

\section{Etapa 7: Importación y exportación de metadatos bibliográficos y de autoridad RDA}

La séptima fase abarca la problemática vinculada con la importación de los metadatos RDA al OPAC de la biblioteca a partir de alguna utilidad o red bibliográfica, o de algún otro OPAC fuente seleccionado por la biblioteca. Las decisiones obvias a ser tomadas en esta etapa constituyen una consecuencia directa de las resoluciones dispuestas en los estadios anteriores, como, por ejemplo, la elaboración de una política de catalogación con RDA, la fijación de una fecha de implementación de RDA y la aceptación 
de metadatos bibliográficos RDA en la medida que estén disponibles en las utilidades bibliográficas desde las que se realiza catalogación derivada. La siguiente acción consiste de continuar aceptando metadatos bibliográficos AACR2 tal como están, o mejorar su calidad con la aplicación de pautas RDA, constituyendo metadatos híbridos, tal como se mencionó anteriormente. La abundancia y la presencia mayoritaria de metadatos bibliográficos RDA creados por medio de la catalogación original, plantea el desafío de reevaluar esta medida en un futuro cercano. Este proceso de aceptación de metadatos RDA se ve coronado con el diseño de una lista de control (checklist) acorde a RDA y MARC21, elaborada como parte de la política de catalogación de la biblioteca a nivel local y dirigida a guiar las operaciones de catalogación por copia del personal (Maurer y Panchyshyn, 2014).

\section{Etapa 8: Aplicación de RDA a los metadatos de autoridad}

El octavo ítem más que constituir una etapa de implementación en sí, quiere llamar la atención sobre el hecho de que RDA incorpora el trabajo de autoridad y los metadatos de autoridad dentro de sus pautas. El capítulo 6 de la Sección 2 de la versión original de RDA se focaliza en establecer datos de autoridad, incluyendo un punto de acceso autorizado. Una vez que la obra o expresión se encuentra establecida en un metadato de autoridad, el punto de acceso autorizado creado como parte del trabajo de autoridad, puede usarse en metadatos bibliográficos. En la actualidad, estos datos emanados de RDA se codifican en metadatos de autoridad MARC21. Los datos de autoridad establecidos por RDA se rigen por el objetivo costo y eficiencia, que equivale al principio de economía enunciado por los PIC. En tal sentido, aunque el control de autoridad tiene la fama de ser caro, el imperativo marca que las bibliotecas seleccionen aquellas opciones más económicas disponibles en relación a su seguimiento y aplicación (Joudrey, Taylor y Miller, 2015; IFLA Cataloguing Section y IFLA Meeting of Experts on an International Cataloguing Code, 2016).

\section{Organización de la información y empleo de RDA en diversos lugares del mundo}

Las bibliotecas nacionales adquieren un rol central y predominante en relación con la conducción de la implementación de RDA, en diferentes lugares del mundo, siendo la realización y la publicación de los resultados del U.S. RDA Test, desarrollado en Estados Unidos, un primer paso fundacional en esta dirección. El U.S. RDA Test Coordinating Committee recomienda a partir de la publicación del informe, el 1 de julio de 2011, no implementar RDA en Estados Unidos antes del 1 de enero de 2013 (U.S. RDA Test Coordinating Committee, 2011). Finalmente, LC implementó RDA el 31 de marzo de 2013 (Maurer y Panchyshyn, 2014; Tosaka y Park, 2013). El resto de las bibliotecas nacionales de los países anglófonos no llevan a cabo ningún test de alcance nacional e incorporan los resultados que emergen de la prueba de RDA realizada por las bibliotecas norteamericanas, realizando la implementación el mismo año que LC en Estados Unidos o en años subsiguientes (Joudrey, Taylor y Miller, 2015). Durante 2010, las bibliotecas nacionales y las asociaciones profesionales de Gran Bretaña, de Australia, de Nueva Zelanda y de Canadá llevan a cabo encuestas dirigidas a contar con una visión general del estado de situación de RDA con respecto a las bibliotecas y a los profesionales en cada uno de tales países. También, en los años subsiguientes, la Biblioteca Nacional de España (BNE) y la Biblioteca Nacional Mariano Moreno de la República Argentina (BNMM), desarrollan encuestas sobre RDA en las bibliotecas inmersas dentro de ambos países. A continuación, se enumeran los principales resultados de algunas de estas encuestas que son de particular interés para esta investigación.

El Australian Committee on Cataloging (ACOC) y la National Library of New Zealand (NLNZ), realizan una encuesta sobre RDA en marzo de 2010 en base a un conjunto 
de preguntas similares. En Nueva Zelanda, gran parte de los encuestados (cerca del 70\%) manifiestan niveles limitados o bajos de conocimiento sobre RDA. Esta situación conduce directamente a elegir un entrenamiento de RDA con una fuerte impronta práctica, abarcando temas como la catalogación con RDA, los cambios AACR2-RDA, la relación MARC21-RDA y el uso del RDA Toolkit. Asimismo, menos del $30 \%$ de los encuestados informan sentirse capaces de aplicar o comprender RDA, siendo las cuestiones operativas y de uso del estándar, las áreas predominantes de interés para el entrenamiento (Todd, Stretton y Stewart, 2010). NLNZ abarca en su encuesta bibliotecas especiales, públicas, de college y politécnicos, escolares, universitarias y nacional. La mayoría de las bibliotecas encuestadas se encuentran agrupadas en dos redes bibliográficas principales, a la vez que utilizan una amplia variedad de SIGB. Cuando se consulta a las bibliotecas si planifican usar RDA, responden afirmativamente el $18 \%$ de ellas, mientras que el $43 \%$ supedita la decisión al hecho de que inicialmente lo lleve a cabo NLNZ. Finalizan las respuestas negativas (4\%) y de inseguridad (35\%). Puede considerarse que el $61 \%$ planea aplicar RDA, en la medida que NLNZ utilice el estándar. En consonancia con los guarismos acerca de la gran proporción de bibliotecas neozelandesas que no utilizan actualmente MARC21 en sus sistemas automatizados, los que ascienden al 45\%, NLNZ considera que los cursos de entrenamiento deben incorporar y formalizar la enseñanza de MARC21, en tanto esquema de codificación predominante aplicado con RDA (Todd, Stretton y Stewart, 2010).

La implementación completa de RDA en Canadá requiere la traducción del estándar al francés, constituyendo un pre-requisito fundamental disponer de esta versión para su implementación en las bibliotecas canadienses que ofrecen catálogos bilingües o exclusivamente en lengua francesa. En este país, se manifiesta una situación dual con la implementación de RDA en sus regiones inglesa y francesa. La adopción de RDA en las bibliotecas canadienses de habla inglesa se da en forma completa ( $10 \%)$, de manera parcial (50\%) y sin adopción (35\%). El 60\% de las bibliotecas brinda un entrenamiento básico de RDA para profesionales de sectores de referencia, adquisiciones, sistemas y administración de bibliotecas. Las bibliotecas académicas de mayor magnitud adop$\tan$ mayoritariamente RDA en un $75 \%$ de ellas (Cross et al, 2014). Como se refirió anteriormente, la versión en inglés de RDA se publica en 2010, mientras que, para la comunidad de catalogación de habla francesa, tanto la finalización de la traducción como la implementación de RDA coinciden durante el año 2013. En tal sentido, la fase inicial del entrenamiento de RDA en francés, emerge del proyecto de traducción del estándar. Los módulos de aprendizaje originalmente elaborados en inglés se traducen y adaptan a la lengua francesa, abarcando contenidos como el trasfondo de RDA, el RDA Toolkit, los modelos conceptuales FR y el formato MARC21, entre los temas principales. La comunidad de catalogación no cuenta inicialmente con documentación de entrenamiento en francés, en la medida que Canadá es el primer país en conducir la implementación de RDA en dicha lengua (Cross et al, 2014).

Los postulados enunciados más arriba pueden servir de parámetros para ser considerados en la experiencia de implementación de RDA que se lleve a cabo en Argentina, como el hecho de rubricar la posición hegemónica que debería ocupar la BNMM en Argentina con respecto a RDA, emulando el rol de NLNZ, y de otras bibliotecas nacionales del mundo, en la conducción del proceso de implementación de RDA para el resto de las bibliotecas del país. En sintonía con la función central que las bibliotecas nacionales alrededor del mundo han tomado en relación a la inserción de RDA, la BNMM de Argentina condujo diversas acciones iniciales vinculadas con la implantación de RDA en Argentina, como el proyecto cooperativo, junto con las bibliotecas nacionales de España, Colombia y Chile y LC, bajo la órbita de la Asociación de Bibliotecas Nacionales de Iberoamérica (ABINIA), de traducción al español del material de entrenamiento de RDA elaborado por 
LC. Esta línea de acción se asimila con el inicio de la fase de entrenamiento en RDA, como primer paso a ser seguido dentro del proceso de implementación del estándar en Argentina (Barber y Salta, 2017).

La encuesta desarrollada por la BNE, en España, en 2014, muestra que una amplia mayoría de las bibliotecas ( $83 \%$ ) aún no han resuelto nada respecto a la implementación de RDA en dicho país, mientras que el $14 \%$ se muestra decidido a llevarla a cabo y el $3 \%$ es afín a la no implantación del nuevo estándar (Biblioteca Nacional de España, 2014). El grupo de bibliotecas que ha tomado la decisión de implementar RDA (14\%), señala el bienio 2016/2017 como fecha elegida. Con respuestas minoritarias, se encuentran las alternativas de aguardar que se publique la traducción al español-la que aparece en 2015 en el RDA Toolkit y en 2016 en formato impreso-y seguir la agenda que indique la $\mathrm{BNE}$, ambas con valores cercanos al 10\% (Biblioteca Nacional de España, 2014). En cuanto al grupo de bibliotecas que aún no han resuelto la implementación de RDA (83\%), un 60\% de ellas sostiene reconsiderar la decisión en caso de que la BNE decida adoptar el nuevo estándar, mientras que en el grupo de bibliotecas que decide no implementar RDA (3\%), consideran proseguir con las reglas de catalogación actuales, no estando dispuestas cerca del $75 \%$ de ellas a reveer su decisión en el caso de que BNE decida implementar el estándar (Biblioteca Nacional de España, 2014).

La BNMM de Argentina realiza una encuesta, en 2015, donde una mayoría abrumadora de las bibliotecas encuestadas (cerca del 94\%) respondió que aún no había decidido nada acerca de la implementación de RDA, seguido por un pequeño grupo (cercano al $4 \%$ ) que había decidido implementar el nuevo estándar. Finalmente, un guarismo mínimo (de más del 1\%) afirmaba no implementar el nuevo estándar. Dentro del grupo de bibliotecas que aún no decidió implementar RDA (cerca del 94\%), el 40\% sostuvo que la determinación que tome BNMM acerca de RDA puede condicionar su decisión inicial (Biblioteca Nacional Mariano Moreno de la República Argentina, 2015). Finalmente, el Grupo de Trabajo RDA para América Latina y el Caribe, conduce una encuesta sobre el estado de situación de RDA, durante 2019, en las bibliotecas nacionales de Iberoamérica, vinculadas con ABINIA. Las bibliotecas encuestadas manifiestan que utilizan AACR2 (31\%), RDA (23\%) y la combinación de estándares -que denota el grado de hibridez existente-AACR2-RDA (23\%), AACR2-ISBD (cerca del 15\%) e ISBD-RDA (más del 7\%). En relación al estado de implementación de RDA en las bibliotecas nacionales de Iberoamérica, la mayoría de ellas (más del 38\%) afirmó estar implementando RDA y un guarismo similar (cercano al 38 \%) sostuvo que piensa en implementar RDA en el futuro. Continúan aquellas bibliotecas nacionales que expresaron ya haber implementado RDA (más del 15\%) y aquellas que no piensan implementar el nuevo estándar (cerca del 8\%) (Barber, 2019).

\section{Marco teórico, objetivos y método de la investigación}

El marco teórico de la investigación se encuadra en las categorizaciones de capital, habitus, campo y sentido práctico esgrimidas por Bourdieu, siendo de especial interés los aspectos relativos a la implementación del estándar RDA en el continuum de las prácticas de la organización de la información seguidas en las bibliotecas argentinas. El control bibliográfico u organización de la información, donde RDA opera como código de catalogación de alcance internacional, se asimila con la noción bourdiana de campo de fuerzas dotado de una estructura, así como de campo de luchas para conformar o transformar ese campo de fuerzas (Salta, 2014). La estructura del campo consiste en un estado de relación de fuerzas entre agentes que toman parte de la lucha, en pos de la distribución de un capital específico, variable, temporal y espacialmente constituido, y se instituye en condición de funcionamiento del campo, en tanto hace que los agentes 
concurran, compitan y luchen (Bourdieu, 2000; Bourdieu, 2003). Las prácticas de los agentes se vislumbran, de esta manera, como producto del encuentro de dos historias ajustadas entre sí. Una de ellas, comportando una objetividad de primer orden presente en la propia estructura del campo, que se advierte en los objetos e instrumentos técnicos y en los textos. La otra historia está incorporada como disposiciones, donde lo social se encuentra en la subjetividad, en las mentes de los individuos, constituyendo una objetividad de segundo orden o una subjetividad objetiva. Consiste de formas de hacer, de pensar, de sentir y de juzgar de los individuos sociales que están contenidas en el habitus, que se presenta como un sistema de clasificación, de esquemas mentales y corporales (Bourdieu, 2007; Tovillas, 2010). El habitus suele manifestarse en los exámenes orales, en las exposiciones de seminarios, en los contactos con los demás, en las posturas corporales, haciendo que los agentes caigan en la práctica que es suya, que no eligen libremente ni los coacciona mecánicamente (Bourdieu, 2000; Bourdieu, 2003). El sentido práctico es un concepto articulador de las nociones de campo y habitus, por el que los agentes actúan de forma inmediata y natural en las situaciones sociales, sin realizar una reflexión tácita consciente. El sentido práctico dispuesto por un sujeto en un campo determinado, implica que actúe regularmente como se debe, que haga lo que tiene que hacer y produzca anticipaciones correctas (Bourdieu, 2007; Tovillas, 2010). En el contexto de esta investigación, las nociones de habitus y de capital refieren a la formación académica y las realizaciones materiales del tipo de artículos, monografías, ponencias en conferencias, congresos, etc., de los agentes, tanto en el aspecto teórico y práctico del control bibliográfico y en lo relativo a los modelos lógicos emergentes del universo bibliográfico, con particular énfasis en la catalogación, la codificación y el control de autoridad de los recursos de información. Asimismo, ambas nociones conceptuales abarcan el producto de dicho control bibliográfico - es decir, los registros o metadatos bibliográficos- obtenido por la mediación de lenguajes bibliográficos, del tipo de estándares de descripción y acceso y formatos de codificación.

Budd (2003) afirma que las bibliotecas participan y contribuyen con la producción cultural de dos maneras discernibles. En la primera de ellas, reciben la visita del público que busca consumir los productos culturales que coleccionan, a la vez que, recursivamente, tales productos se ponen disponibles, en la medida que se crean y distribuyen, para alcanzar al público. La comunidad o el público al que sirven los distintos tipos de bibliotecas, constituye una noción cargada de poder simbólico. En tal sentido, el concepto de comunidad no consiste en un agregado, yuxtaposición o reunión libre de personas en un lugar donde se brinda el acceso a bienes simbólicos, sino que las bibliotecas son actores centrales y activos en la construcción de los deseos y expectativas de tales comunidades. Las bibliotecas adquieren, por distintas modalidades y de forma intencional, ciertos bienes simbólicos, situación que implica su participación indirecta en la producción cultural, en tanto utilizan modos de producción cultural - como revisiones, anuncios, etc.- para llevar a cabo tal elección. Por consiguiente, la organización de la información llevada a cabo por las bibliotecas corresponde al campo de la producción cultural. Esta pertenencia puede imbricarse en el pensamiento y la acción de los bibliotecarios, o puede permanecer inconsciente sin la debida reflexión formal e intencional de los servicios o procesos técnicos desarrollados por la biblioteca y su impacto sobre los usuarios que acceden a ella para hacerse de bienes simbólicos (Budd, 2003).

\section{Objetivos, método y procedimientos de la investigación}

La investigación presenta objetivos de naturaleza principal y secundaria. El objetivo principal consiste en analizar las características, las modalidades y las vicisitudes particulares que entraña y adquiere el proceso de implementación del estándar RDA en las bibliotecas de la República Argentina. Los objetivos secundarios tratan, en 
primer lugar, con comprender la incidencia de las prácticas de la organización de la información llevadas a cabo por las bibliotecas argentinas. En segundo lugar, buscan describir el ambiente híbrido y transicional del control bibliográfico, signado por el uso de un estándar novedoso - como RDA - en consonancia con estándares preexistentes - como AACR2, ISBD y MARC21.

Esta investigación se encuadra en un abordaje cualitativo que aplica los postulados de vigilancia epistemológica y de objetivación del sujeto de la objetivación, establecidos por Bourdieu (Bourdieu, Chamboredon y Passeron, 2011). El investigador encuentra en la vigilancia epistemológica, como ejercicio constante, un instrumento para examinar las condiciones y los límites de validez del uso de técnicas y conceptos en la investigación científica, desterrando la comodidad de su aplicación automática a la par de reflexionar sobre toda operación en sí misma y en función del caso particular (Bourdieu, Chamboredon y Passeron, 2011). En tanto y en cuanto el investigador forma parte del mundo que intenta objetivar, dando lugar a lo oculto - es decir, lo que se refugia en la mirada del científicodesarrolla el procedimiento de objetivar al sujeto de la objetivación, tarea que se realiza en tres niveles (Bourdieu, 2003; Tovillas, 2010). En primer lugar, se objetiva la posición del sujeto de objetivación en el espacio social, enfatizando su origen y trayectoria, su pertenencia, sus adhesiones sociales, etc. En segundo término, se establece la estructura objetiva de relaciones entre las posiciones ocupadas por los agentes que compiten dentro del campo sus pensamientos, sus creencias y evidencias compartidas, sus rituales y consagraciones, las presiones de publicación de resultados, la censura, los presupuestos inscritos en la historia colectiva de la especialidad, entre los aspectos significativos a considerar. En tercer lugar, se analizan los habitus de los agentes, los diferentes sistemas de disposiciones adquiridos por medio de interiorizar un tipo determinado de condiciones sociales y económicas, es decir, la forma en que se valorizan las posiciones y las recompensas ofrecidas por el campo.

Bourdieu, Chamboredon y Passeron (2011) establecen cuatro principios fundamentales que estructuran el habitus del sociólogo. En primer término, la ruptura con el sentido común, que el científico social entabla contra las ideas prefabricadas, naturalizadas en las conciencias individuales. Continua el principio de no conciencia, por el que las representaciones sociales y los discursos propios de los agentes deben generar prudencia en el investigador, en la medida que constituyen elementos a ser explicados, antes que constituir una explicación que cierre el análisis sociológico. En tercer lugar, se confiere primacía a las relaciones en el análisis sociológico, en tanto el mundo social es una realidad social externa e independiente, que inserta a individuos en condiciones y en posiciones sociales dentro de un espacio social estructurado. Finalmente, se plantea la objeción de explicaciones de tipo transhistóricas o trascendentales de la naturaleza humana, derivadas de principios biológicos, teleológicos, psicológicos, etc.

Esta investigación planea utilizar los procedimientos de observación, lectura y análisis de las fuentes vinculadas con la delimitación del objeto de estudio, así como la elaboración de una encuesta de tipo autoadministrada, es decir, sin participación del encuestador, con la intermediación de un cuestionario semiestructurado, a ser enviado a bibliotecarios profesionales que se desenvuelvan en distintas bibliotecas de la República Argentina. Con la finalidad de proporcionar apreciaciones más sustanciales y ricas acerca de la temática de investigación, se plantea, en sintonía con la metodología aplicada por Park y Tosaka (2015), la posibilidad de llevar a cabo entrevistas a pequeña escala con la intención de sacar a relucir aspectos del tópico de investigación que no han emergido conveniente y fácilmente en la encuesta en línea. La aplicación combinada de ambas técnicas de observación, se espera que 
ofrezca una oportunidad factible y viable para reunir datos organizados, en profundidad y confiables acerca de las experiencias y las necesidades de los actores en relación a sus prácticas en la organización de la información.

\section{A modo de conclusión}

El interrogante primordial de la investigación enunciada radica en reflexionar acerca de las prácticas de organización de la información seguidas en las bibliotecas argentinas, a la par de su imbricación, asimilación y concurrencia con el proceso de implementación del nuevo estándar de catalogación RDA en el país. La hipótesis de la investigación consiste en pensar en la existencia de una cantidad creciente de metadatos bibliográficos y de autoridad elaborados en base a AACR2 y a RDA, o que integren elementos de datos extraídos de ambos estándares de descripción y acceso en forma de metadatos mezclados, todos ellos codificados con MARC21. Este ambiente transicional e híbrido de organización de información existente principalmente en la Biblioteca Nacional del país, y por extensión en la mayor parte de las bibliotecas nacionales de lengua española, puede estar disponible y ser aprovechado por el resto de las bibliotecas argentinas, por medio del desarrollo de procedimientos de catalogación por copia o derivada de los metadatos bibliográficos y de autoridad. La descarga e incorporación creciente de metadatos bibliográficos y de autoridad RDA, así como de metadatos AACR2 a los que se adicionen elementos extraídos de RDA, permitirá la incorporación y asimilación gradual de las pautas y los elementos del nuevo estándar de descripción y acceso de parte del personal de las bibliotecas argentinas. La unidad de análisis de la investigación estará constituida por los bibliotecarios en ejercicio profesional en los diferentes tipos de bibliotecas existentes en la República Argentina. La población definida y abarcada por la investigación, será representada por medio de un muestreo no aleatorio, logrado mediante la distribución del formulario de la encuesta a listas de discusión profesional y a los correos electrónicos de bibliotecarios profesionales en ejercicio en Argentina.

El diseño de la investigación, delinea una serie de propiedades fundamentales relacionadas con la unidad de análisis de la investigación, las que van a representar las variables de los casos bajo estudio. La primera de tales propiedades, se vincula al grado de familiaridad y conocimiento de RDA de parte de la población estudiada. Particularmente, interesa saber el nivel de conocimiento y con qué espectro de habilidades y competencias profesionales relacionadas con RDA cuentan los bibliotecarios argentinos. La siguiente cualidad está dirigida a conocer él o los estándares de descripción y acceso y el o los formatos de codificación utilizados en las prácticas de organización de información seguidas por las bibliotecas del país. En el caso particular de RDA, incumbe conocer si se utiliza la versión impresa o la versión en línea dentro del RDA Toolkit. La tercera característica trata con la indagación acerca de las necesidades más apremiantes detectadas para lograr la incorporación de RDA como estándar que gobierne las prácticas de organización de información seguidas por las bibliotecas argentinas. La cuarta propiedad se relaciona con la gestión del proceso de implementación de RDA en la Argentina, enfatizando la dirección de la implantación del nuevo estándar sobre la Biblioteca Nacional -a la usanza de lo ocurrido en otros lugares del mundo-y tratando de elucidar los roles podrían corresponderles a los distintos agentes del ámbito bibliotecario a nivel local, regional y nacional. En lo concerniente al entrenamiento en RDA, el quinto atributo bajo consideración, que comporta una de las etapas iniciales y principales de la implementación de RDA, interesa saber qué consecuencias acarrea el hecho de que los recursos de entrenamiento existentes en la actualidad sobre este estándar se encuentren casi exclusivamente en inglés. Siguiendo con la capacitación en el uso del nuevo estándar de catalogación, es crucial discernir que modalidades de entrenamiento son más aconsejables para 
aplicar en la Argentina, considerando los aspectos relativos al estado de conocimiento sobre RDA, la duración de las sesiones y los canales de diseminación preferidos y posibles para su aplicación. Finalmente, cuáles serían los contenidos que debería contemplar el entrenamiento en RDA, considerando el grado de incidencia que las prácticas de organización de información de las bibliotecas argentinas tienen en relación al adiestramiento profesional sobre el nuevo estándar. El sexto rasgo explora las modalidades de catalogación y de control de autoridad imperantes en las prácticas de la organización de la información desarrolladas por los bibliotecarios argentinos. Interesa, particularmente, lo relativo a la adopción de RDA en las distintas modalidades de catalogación (original, por copia, en publicación) junto con las razones que fundamentan tal decisión, los tipos de recursos de información descriptos con RDA bajo cada modalidad de catalogación y el agregado, revisión o eliminación de elementos de RDA o de AACR2 sobre los metadatos bibliográficos y de autoridad en la catalogación por copia. La séptima propiedad trata con la integración o hibridación de elementos en metadatos bibliográficos y de autoridad extraídos de los estándares AACR2 y RDA, siendo clave la indagación de ciertos aspectos fundamentales relacionados con este tópico. En primer término, la elaboración de políticas y de procedimientos de catalogación con $\mathrm{RDA}$, enfatizando las fuentes preexistentes que servirían de base para la determinación de que documentos normativos deberían tomar en cuenta las bibliotecas argentinas para enfrentar la transición entre AACR2 y RDA. Este aspecto debe prestar especial atención a las tradiciones imperantes en las prácticas de organización de la información seguidas por las bibliotecas argentinas y a las particularidades culturales, lingüísticas, etc., propias del país. En segundo término, estimar el grado de impacto de estas políticas y procedimientos sobre los procesos de catalogación imperantes en las bibliotecas argentinas. En particular, es importante poner bajo consideración la posibilidad de re-catalogar la totalidad de metadatos bibliográficos existentes elaborados con AACR2 hacia RDA. Finalmente, el octavo atributo plantea la necesidad de verificar en qué grado deberían seguirse los pasos y los resultados obtenidos en los procesos de implementación realizados en diferentes lugares del mundo, en tanto factores que afecten y condicionen las características particulares de las prácticas de la organización de la información encontradas en las bibliotecas de la República Argentina. 


\section{Q Referencias bibliográficas}

》Barber, Elsa. E. 2019. Acciones desarrolladas por el Grupo de Trabajo RDA para América Latina y el Caribe: periodo octubre de 2018 a octubre de 2019. 10 p. Trabajo presentado al XXX Asamblea General de ABINIA, realizado en Biblioteca Nacional, Lima, Perú del 14 al 18 de octubre de 2019.

»Barber, Elsa. E. y Gerardo Salta. 2017. Aproximaciones e intervenciones de la Biblioteca Nacional Mariano Moreno de la República Argentina con respecto a RDA: 2012-2017. En Información, Cultura y Sociedad. No. 37, 41-58. <https://doi. org/10.34096/ics.i37.3750>

»Behrens, Renate, Christine Frodly Renate Polak-Bennemann. 2014. The Adoption of RDA in the German-Speaking Countries. En Cataloging \& Classification Quarterly. Vol. 52 no. 6/7, 688-703. <https://doi.org/10.1080/01639374.2014.882872>

» Biblioteca Nacional de España. 2014. Encuesta sobre RDA: Mayo-Junio 2014. <http://www.bne.es/es/Inicio/Perfiles/Bibliotecarios/Procesos-tecnicos/NormasInternacionales/RDA/RDA-BNE/> [Consulta: 17 agosto 2021]

»Biblioteca Nacional Mariano Moreno de la República Argentina. 2015. Encuesta sobre RDA. MS. $10 \mathrm{p}$.

»Bourdieu, Pierre. 2000. Cosas dichas. Barcelona: Gedisa.

"Bourdieu, Pierre. 2003. El oficio de científico: ciencia de la ciencia y reflexividad. Barcelona: Anagrama.

»Bourdieu, Pierre. 2007. El sentido práctico. México, D.F.: Siglo XXI.

"Bourdieu, Pierre, Jean-Claude Chamboredon y Jean-Claude Passeron. 2011. El oficio de sociólogo. Buenos Aires: Siglo XXI.

»Budd, John M. 2003. The library, praxis, and symbolic power. En Library Quarterly. Vol. 73, no. 1. <https://doi.org/10.1086/603373>

»Choi, Kathy, Haslinda M. Yusof y Fauziah Ibrahim. 2014. RDA: National Library Board Singapore's learning Journey. En Cataloging \& Classification Quarterly. Vol. 52, no. 6/7, 608-620. <https://doi.org/10.1080/01639374.2014.891165>

"Cronin, Christopher. 2011. From Testing to Implementation: Managing Full-Scale RDA Adoption at the University of Chicago. En Cataloging \& Classification Quarterly. Vol. 49, no. 7/8, 626-646. <https://doi.org/10.108o/01639374.2011.616263>

"Cross, Emma, Sue Andrews, Trina Grover, Chris Oliver y Pat Riva. 2014. In the Company of My Peers: Implementation of RDA in Canada. En Cataloging \& Classification Quarterly. Vol. 52, no. 6/7, 747-774. <https://doi.or g/10.1080/01639374.2014.899535>

»El-Sherbini, Magda 2013. RDA: Strategies for Implementation. Chicago, II.: ALA Editions.

"Hanson, Eric y Bonnie Parks. 2013. RDA Training and Implementation at the University of Chicago: An Interview with Christopher Cronin. En Serials Review. Vol. 39, no. 2, 136-140. <https://doi.org/10.108o/o0987913.2013.10765507> 
" Hitchens, Alison y Ellen Symons. 2009. Preparing Catalogers for RDA Training. En Cataloging \& Classification Quarterly. Vol 47, no. 8, 691-707. <https://doi. org/10.1080/01639370903203234>

» IFLA. Sección de Catalogación e IFLA. Reuniones de Expertos sobre un Código Internacional de Catalogación. 2016. Declaración de Principios Internacionales de Catalogación (PIC). Edición 2016. <https://www.ifla.org/files/assets/cataloguing/icp/icp_2016-es.pdf> [Consulta: 30 agosto 2021]

» Joudrey, Daniel N., Arlene G. Taylor y David P. Miller. 2015. Introduction to Cataloging and Classification. 11th ed. Santa Barbara, CA.: Libraries Unlimited.

» Joudrey, Daniel. N., Arlene. G. Taylor y Katherine M. Wisser. 2018. Organization of information. 4th ed. Santa Barbara, CA.: Libraries Unlimited.

» Kuhagen, Judith A. 2011. Training for the U.S. RDA Test. En Cataloging \& Classification Quarterly. Vol. 49, no. 7-8, 572-581. <https://doi.or g/10.1080/01639374.2011.616924>

» Maurer, Margaret B. y Roman S. Panchyshyn. 2014. Understanding the Why: A Case Study in Managing the RDA Implementation. En Cataloging \& Classification Quarterly. Vol.52, no.3, 259-284. <https://doi.org/10.1080/01639374.2013.859644>

" OCLC. 2019. Declaración de Políticas de RDA de OCLC. <https://www.oclc.org/ es/rda/new-policy.html> [Consulta: 28 agosto 2021]

" Oliver, Chris. 2010. Introducing RDA: a guide to the basics. Chicago. Il.: American Library Association.

»Park, Jung-ran y Yuji Tosaka. 2015. RDA Implementation and Training Issues across United States Academic Libraries: An In-Depth E-Mail Interview Study. En Journal of Education for Library and Information Science. Vol. 56, no. 3, 252-266. $<10.12783$ /issn.2328-2967/56/3/6>

»RDA Steering Committee. 2020. About RDA. <http://www.rda-rsc.org/content/ about-rda> [Consulta: 20 agosto 2021]

» RDA Toolkit. 2021. <https://access.rdatoolkit.org/> [Consulta: 17 septiembre 2021]

"Salta, Gerardo. 2014. La estructura, el diseño y el contenido de RDA: La pugna entre diferentes configuraciones y ordenamientos. Lanús: UNLa, 2014. 215 p. Tesis de Maestría. <http://www.repositoriojmr.unla.edu.ar/descarga/Tesis/MAMIC/035576_Salta.pdf> [Consulta: 17 septiembre 2021]

"Salta, Gerardo. 2016. Las contiendas alrededor de la configuración y el ordenamiento en RDA: las discusiones y deliberaciones sobre los borradores publicados entre 2004 y 2008. En Información, cultura y sociedad. No. 35, 11-30. <http:// revistascientificas.filo.uba.ar/index.php/ICS/article/view/2438> [Consulta: 17 septiembre 2021]

"Salta, Gerardo. 2020. La implementación de RDA en Argentina: Características, modalidades y vicisitudes particulares en las prácticas del control bibliográfico seguidas en las bibliotecas. MS. $30 \mathrm{p}$.

»Todd, Chris, Charlotte Stretton y Janess Stewart. 2010. RDA Training Needs Survey (New Zealand). National Library of New Zealand. <http://nznuccataloguing. pbworks.com/w/page/26673174/RDA\%20training\%2oneeds\%2oanalysis> [Consulta: 17 septiembre 2021] 
»Tosaka, Yuji y Jung-ran Park. 2013. RDA: Resource Description \& Access: a survey of the current state of the art. En Journal of The American Society for Information Science \& Technology. Vol. 64, no. 4, 651-662. <https://doi.org/10.1002/ asi.22825>

»Tovillas, Pablo. 2010. Pierre Bourdieu: una fuga por lo cultural. Buenos Aires: Quadrata, Biblioteca Nacional.

" U.S. RDA Test Coordinating Committee. 2011. Report and recommendations: 9 May 2011, revised for public release 20 June 2011. <http://www.loc.gov/bibliographic-future/rda/source/rdatesting-finalreport-20june2011.pdf> [Consulta: 30 agosto 2021]

"Welsh, Anne y Katharine Whaite. 2012. Our Hybrid History and its lessons for today. En Catalogue \& Index. Vol. 169, 5-9. 